\title{
HLA-DR2-restricted responses to proteolipid protein 95-116 peptide cause autoimmune encephalitis in transgenic mice
}

\author{
Kazuyuki Kawamura, ${ }^{1}$ Takashi Yamamura, ${ }^{1}$ Kazumasa Yokoyama, ${ }^{1}$ De-Hua Chui, ${ }^{1}$ \\ Yoshinori Fukui, ${ }^{2}$ Takehiko Sasazuki, ${ }^{2}$ Hidetoshi Inoko, ${ }^{3}$ Chella S. David, ${ }^{4}$ \\ and Takeshi Tabira ${ }^{1}$ \\ ${ }^{1}$ Department of Demyelinating Disease and Aging, National Institute of Neuroscience, \\ National Center of Neurology and Psychiatry, Kodaira, Tokyo 187-8502, Japan \\ ${ }^{2}$ Department of Genetics, Medical Institute of Bioregulation, Kyushu University, and \\ Core Research for Evolutional Science and Technology (CREST), Japan Science and Technology Corporation, \\ Fukuoka 812-8582, Japan \\ ${ }^{3}$ Department of Molecular Life Science, Tokai University School of Medicine, Kanagawa 259-1100, Japan \\ ${ }^{4}$ Department of Immunology, Mayo Clinic and Medical School, Rochester, Minnesota 55905, USA \\ Address correspondence to: Takeshi Tabira, Department of Demyelinating Disease and Aging, National Institute of \\ Neuroscience, National Center of Neurology and Psychiatry, 4-1-1 Ogawahigashi, Kodaira, Tokyo 187-8502, Japan. \\ Phone: 81-42-341-2711; Fax: 81-42-346-1747; E-mail: tabira@ncnp.go.jp.
}

Received for publication September 13, 1999, and accepted in revised form February 1, 2000.

In multiple sclerosis (MS) patients who carry the Class II major histocompatibility (MHC) type HLA$D R 2$, T cells specific for amino acids 95-116 in the proteolipid protein (PLP) are activated and clonally expanded. However, it remains unclear whether these autoreactive $T$ cells play a pathogenic role or, rather, protect against the central nervous system (CNS) damage. We have addressed this issue, using mice transgenic for the human MHC class II region carrying the HLA-DR2 $(D R B 1 * 1502)$ haplotype. After stimulating cultured lymph node cells repeatedly with PLP95-116, we generated 2 HLADR2-restricted, PLP95-116-specific T-cell lines (TCLs) from the transgenic mice immunized with this portion of PLP. The TCLs were $\mathrm{CD}^{+}$and produced T-helper 1 (Th1) cytokines in response to the peptide. These TCLs were adoptively transferred into $R A G-2^{-/-}$mice expressing HLA-DR2 (DRB1*1502) molecules. Mice receiving 1 of the TCLs developed a neurological disorder manifested ataxic movement without apparent paresis on day 3 , 4 , or 5 after cell transfer. Histological examination revealed inflammatory foci primarily restricted to the cerebrum and cerebellum, in association with scattered demyelinating lesions in the deep cerebral cortex. These results support a pathogenic role for PLP95116-specific T cells in HLA-DR2 ${ }^{+}$MS patients, and shed light on the possible correlation between autoimmune target epitope and disease phenotype in human CNS autoimmune diseases.

J. Clin. Invest. 105:977-984 (2000).

\section{Introduction}

Multiple sclerosis (MS) is a chronic inflammatory disease of the central nervous system (CNS) that is histologically characterized by focal mononuclear cell infiltration with a varying extent of demyelination $(1,2)$. It is postulated that autoimmune $\mathrm{T}$ cells recognizing myelin autoantigens such as myelin basic protein (MBP) or proteolipid protein (PLP) play a central role in the pathogenesis of MS. This postulate is based on substantial evidence such as increased frequency of activated MBP- or PLP-specific T cells in peripheral blood and cerebrospinal fluid of MS patients (3-6), and significant complementarity-determining region 3 (CDR3) homology between T-cell infiltrates within MS plaques and MBP- or PLP-specific T-cell clones generated from MS patients $(7,8)$. Moreover, previous studies have identified the peptides MBP84-102 and PLP95-116 as immunodominant and possible encephalitogenic epitopes in MS patients with the HLA-DR2 haplotype (9,
10). However, the role of such autoreactive $\mathrm{T}$ cells remains unclear. In fact, it is possible that some (but not all) autoreactive $T$ cells may protect against CNS tissue damage by producing neurotrophic factors (11-13).

To address the issue regarding the function of autoimmune $\mathrm{T}$ cells, we have introduced HLA-DR2 $(D R B 1 * 1502)$ transgenic mice that are able to mount a T-cell response to DR2-related epitopes in the context of HLA-DR2 molecules. To explore the potential pathogenicity of PLP95-116 in HLA-DR2 ${ }^{+}$MS, we challenged the HLA-DR2 $(D R B 1 * 1502)$ transgenic mice with PLP95-116 and generated 2 peptide-specific T-cell lines (TCLs) from the mice. These TCLs were HLADR2-restricted and produced T-helper type 1 (Th1) cytokine in response to the peptide in the presence of mouse or human antigen-presenting cells (APCs) expressing HLA-DR2 (DRB1*1502) molecules. It was remarkable that transfer of 1 of the TCLs induced atypical autoimmune encephalitis involving the deep cere- 
bral cortex in immunodeficient mice generated by mating the HLA-DR2 (DRB1*1502) transgenic mice with recombination activation gene knockout mice (RAG$2^{-/-}$mice) (14). These results support a pathogenic role of PLP95-116-specific T cells in HLA-DR2 ${ }^{+}$MS patients, and indicate a possible correlation between target epitope and disease phenotype in human CNS autoimmune diseases.

\section{Methods}

Mice. C57BL/6J (B6) and RAG-2-/- mice with an $\mathrm{H}-2^{\text {b }}$ background (14) were purchased from CLEA Japan Inc. (Tokyo, Japan) and Taconic Farms (Germantown, New York, USA), respectively. Generation of $D R A$ transgenic mice with a B6 background (DRA-B6) (15) and $D R B 1 * 1502$ transgenic mice with a B10.RQB3 background (DRB1*1502-B10.RQB3) (16) have been described previously. $D R B 1 * 1502$ transgenic mice with a B6 background (DRB1*1502-B6) were obtained by backcrossing the $D R B 1 * 1502-\mathrm{B} 10$.RQB3 mice to $\mathrm{B} 6$ mice for 6 generations. After crossing $D R A$-B6 and $D R B 1 * 1502-B 6$ mice, HLA-DR2 (HLA-DRB1*1502) transgenic mice (HLA-DR2-B6) were selected from their $\mathrm{F}_{1}$ progeny. HLA-DR2-B6 mice were further crossed twice with $R A G-2^{-/-}$mice; $R A G-2^{-/-}$mice expressing HLA-DR2 (DRB1*1502) (HLA-DR2/RAG-2--) were selected from the $\mathrm{N}_{2}$ progeny. Both HLA-DR2-B6 and HLA-DR2/RAG-2-/- mice were identified using PCR as described below. All the mice were kept under specific pathogen-free conditions. Female mice (6-12 weeks of age) heterozygous for the $D R A$ or $D R B 1^{*} 1502$ genes (or both together) were used.

$P C R$. A pair of primers were used for each gene as follows. DRA gene: 5'-GAACATGTGATCATCCAGGCCG-3' and 5'-GATCGGAGTATAGTTGGAGCGC-3'; DRB1 ${ }^{* 1502}$ gene: $5^{\prime}$-CCTAAGAGGGAGTGTCATTTCTTC- $3^{\prime}$ and $5^{\prime}$ TGTGAAGCTCTCACCAACCCC-3'; RAG-2 gene: $5^{\prime}$-CCACCTCTTCGTTATCCAGC- $3^{\prime}$ and $5^{\prime}$-GTCCTCCAAAGAGAACACCC3-'; pMC1 neo/poly(A)+ fragment disrupting endogenous $R A G-2$ gene in $R A G-2^{---}$mice: $5^{\prime}$-TCATCTCACCTTGCTCCTGC- $3^{\prime}$ and $5^{\prime}$-TATGTCCTGATAGCGGTCCG-3'. The genomic DNA extracted from mouse tail tips was amplified by PCR for 30 cycles by using the temperature profile that follows. DRA and $D R B 1 * 1502$ genes: denaturation at $94^{\circ} \mathrm{C}$ for 1 minute, annealing at $66^{\circ} \mathrm{C}$ for 30 seconds, and extension at $72^{\circ} \mathrm{C}$ for 30 seconds. RAG-2 gene and pMC1 neo/poly $(\mathrm{A})^{+}$fragment: denaturation at $94^{\circ} \mathrm{C}$ for 1 minute, annealing at $62^{\circ} \mathrm{C}$ for 30 seconds, and denaturation at $72^{\circ} \mathrm{C}$ for 30 seconds. Amplified PCR products were analyzed on $6 \%$ polyacrylamide gels stained with ethidium bromide.

Reagents. The human PLP95-116 (AVRQIFGDYKTTICGKGLSATV) and MBP143-168 (GVDAQGTLSKIFKLGGRDSRSGSPMA) peptides were purchased from the Peptide Institute, Inc. (Osaka, Japan) and Kurabo Industries, Ltd. (Osaka, Japan), respectively. Incomplete Freund's adjuvant and heat-killed Mycobacterium tuberculosis H37Ra were purchased from Difco
Laboratories (Detroit, Michigan, USA). Recombinant human IL-2 (rhIL-2) was a gift of Shionogi Pharmaceutical Co. Ltd. (Osaka, Japan). Anti-NK1.1 mAb (PK136) was purified from culture supernatants using protein A column chromatography.

Generation and propagation of PLP95-116-specific TCLs. HLA-DR2-B6 mice were immunized in the footpads of both hind feet and in the tail base with $200 \mu \mathrm{L}$ of emulsion containing $200 \mu \mathrm{g}$ of PLP95-116 in incomplete Freund's adjuvant supplemented with $500 \mu \mathrm{g}$ of M. tuberculosis. Ten days after immunization, popliteal and inguinal lymph nodes were removed, and the single-cell suspensions were prepared at $2 \times 10^{6} \mathrm{cells} / \mathrm{mL}$ in complete medium (RPMI-1640 supplemented with $10 \% \mathrm{FCS}, 10 \mathrm{mM}$ HEPES buffer, $5 \times 10^{-5} \mathrm{M} 2$-mercaptoethanol, $2 \mathrm{mM}$ L-glutamine, and $100 \mathrm{U} / \mathrm{mL}$ of penicillin and $100 \mu \mathrm{g} / \mathrm{mL}$ of streptomycin). Two hundred microliters of the suspension was added to 96-well plates and then stimulated with $25 \mu \mathrm{g} / \mathrm{mL}$ of PLP95116 . The cells were fed with the complete medium supplemented with $7.5 \%$ T-cell growth factor medium (supernatant of concanavalin A-stimulated rat splenocyte cultures) every 3 days. An aliquot of each TCL was assayed for antigen specificity on day 14 . The TCLs that were reactive to PLP95-116 were further expanded and were restimulated every 2 weeks with the peptide in the presence of irradiated (35 Gy) syngeneic splenocytes as APCs.

Proliferation and inbibition assays. Splenocytes $(4 \times$ $10^{5} /$ well) from HLA-DR2-B6 or DRA-B6 mice, or PBMCs $\left(2 \times 10^{5} /\right.$ well $)$ from healthy human subjects

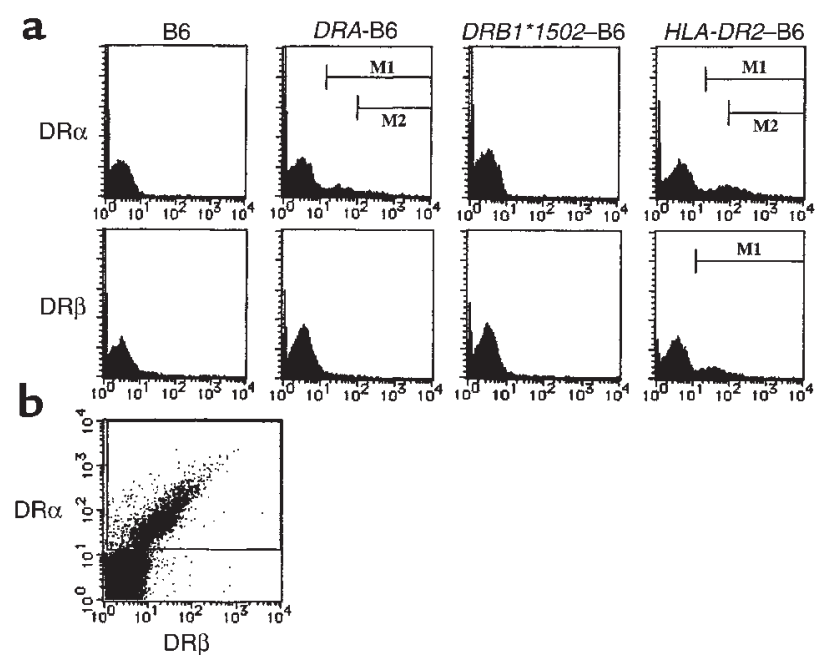

Figure 1

Expression of DR $\alpha / \mathrm{I}-\mathrm{E} \beta^{\mathrm{b}}$ and HLA-DR2 molecules in HLA-DR2-B6 mice. (a) Splenocytes from $\mathrm{B} 6, D R A-\mathrm{B} 6, D R B 1 * 1502-\mathrm{B} 6$, and HLADR2-B6 mice were stained with PE-labeled anti-HLA-DR $\alpha$ (L243) or FITC-labeled anti-HLA-DR $\beta$ (TÜ36) mAb. The bars marked M1 indicate the population that stained significantly with the $\mathrm{mAb}$, whereas $\mathrm{M} 2$ shows the positive population selected on more strict criteria. (b) Splenocytes from HLA-DR2-B6 mice were doubly stained with PE-conjugated anti-HLA-DR $\alpha$ (L243) and FITC-conjugated anti-HLA-DR $\beta$ (TÜ36) mAbs. 


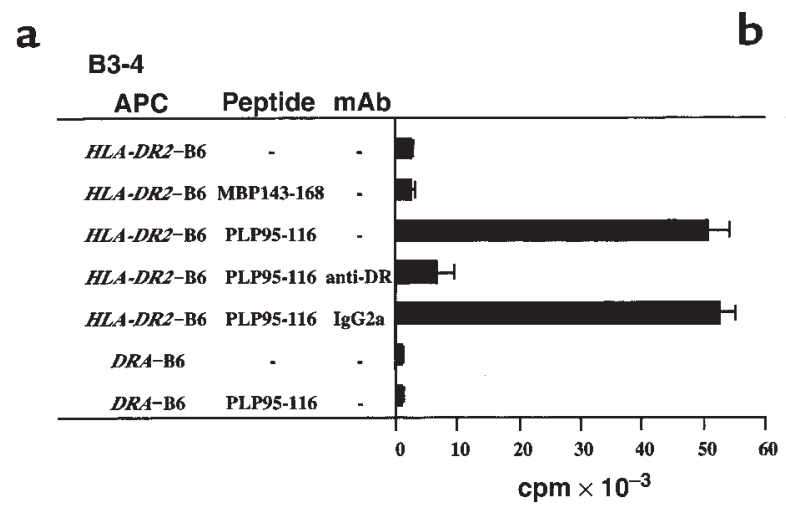

${ }_{\text {B3-23 }}$

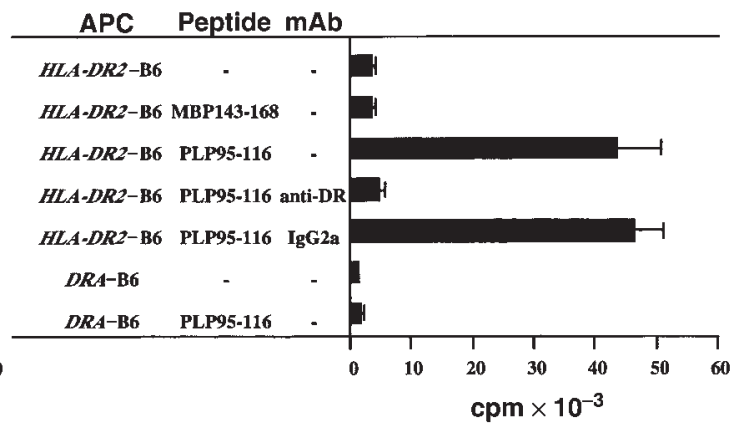

Figure 2

Proliferative response of TCLs B3-4 and B3-23 generated from HLA-DR2-B6 mice immunized with PLP95-116. Peptide-specific proliferation ofTCLs B3-4 (a) and B3-23 (b) were evaluated as described in Methods. By using splenocytes from HLA-DR2-B6 (HLA-DR2 ${ }^{+}$, DR $\alpha /$ I-E $^{\text {b+ }}$, $\mathrm{I}^{-\mathrm{A}^{\mathrm{b}+}}$ ) or DRA-B6 (DR $\left.\alpha / \mathrm{I}-\mathrm{E} \beta^{\mathrm{b}+}, \mathrm{I}-\mathrm{A}^{\mathrm{b}+}\right)$ mice as APCs, T-cell proliferative response to control peptide MBP143-168 or PLP95-116 (25 $\left.\mu \mathrm{g} / \mathrm{mL}\right)$ was measured. Blocking effects of anti-HLA-DR mAb (G46-6) or isotype-matched control IgG2a (G155-178) were also examined as indicated. Data represent mean \pm SD of the cpm obtained by triplicate assays in 4 independent experiments.

designated $\mathrm{MN}(H L A-D R 2[D R B 1 * 1502] / 4)$ and YM ([HLA-DR1]/8) were irradiated (35 Gy for mouse splenocytes and 40 Gy for human PBMCs) and used as APCs. T-line cells $\left(4 \times 10^{4} /\right.$ well $)$ were incubated for 72 hours with or without peptide in the presence of APCs. The cultures were pulsed with $1 \mu \mathrm{Ci}$ of $\left[{ }^{3} \mathrm{H}\right]$ thymidine for the last 16 hours of the incubation. Cell incorporation of $\left[{ }^{3} \mathrm{H}\right]$ thymidine was counted with a 1205 Betaplate counter (Pharmacia, Uppsala, Sweden). Mean cpm of the triplicate cultures was calculated, and TCLs that showed both a stimulation index greater than 3.0 and a change in cpm of more than 500 in the presence of the peptide were defined as peptide-specific. Blocking assays were performed with anti-HLA-DR $\mathrm{mAb}$ (G46-6) or isotype-matched mouse IgG2a (G155-178) added at a final concentration of $10 \mu \mathrm{g} / \mathrm{mL}$.

ELISA. Supernatants were collected from T-cell cultures 48 hours after peptide stimulation. The concentration of cytokines (IFN- $\gamma$, IL-2, TNF- $\alpha$, and IL-4) in the supernatants was measured by sandwich ELISA using pairs of relevant anti-cytokine mAbs according to the protocol recommended by PharMingen (San Diego, California, USA).

Adoptive transfer experiments. HLA-DR2/RAG-2-/- and $R A G-2^{-/-}$mice were intravenously injected with 500 $\mu \mathrm{g}$ of anti-NK1.1 mAb for in vivo depletion of natural killer (NK) cells. The next day, the mice were irradiated ( $3.5 \mathrm{~Gy})$ and injected via tail vein with T-line cells $\left(5 \times 10^{6}\right)$ generated from HLA-DR2-B6 mice. The line cells had been stimulated with PLP95-116 in the presence of syngeneic splenocytes for 3 days. Immediately after cell transfer, $250 \mathrm{ng}$ of pertussis toxin (Seikagaku Kogyo Co. Ltd., Tokyo, Japan) was intravenously injected. All the mice were observed daily for neurological signs.

Flow cytometry. Splenocytes or T-line cells were first incubated with Fc Block (anti-mouse FcR $\gamma \mathrm{II} / \mathrm{III} \mathrm{mAb}$ ) (PharMingen) for 5 minutes, and then incubated for 30 minutes with $\mathrm{mAbs}$ in combination as follows. Spleno- cytes were incubated with either FITC-conjugated anti-HLA-DR $\beta$ (TÜ36) and PE-conjugated anti-HLA$\mathrm{DR} \alpha$ (L243), or FITC-conjugated anti-I-A $\beta^{\mathrm{b}}$ (25-9-17) and PE-conjugated anti-I-A $\alpha^{\mathrm{b}}$ (AF6-12.1). T-line cells were incubated with either FITC-labeled anti-CD4 (RM4-5) and PE-labeled anti-CD8 (53-6.7); FITC-labeled anti-adhesion molecule mAb (anti-LFA-1 [M17/4], antiCD44 [IM7], anti- $\alpha 4$ integrin [M17/4], anti-ICAM-1 [3E2], or anti-L-selectin [MEL-14]) and PE-labeled antiCD4 (RM4-5); or FITC-labeled T-cell receptor (TCR) V $\beta$ chain-specific $\mathrm{mAb}$ (anti-V $\beta 2,-3,-4,-5,-6,-7,-8,-9,-10$, $-11,-12,-13$, or -14 ) and PE-labeled $m A b$ recognizing the common determinant of TCR V $\beta$ chains (H57-597). All mAbs were purchased from PharMingen, except anti-HLA-DR $\alpha$ (Becton Dickinson Immunocytometry Systems, Mountain View, California, USA). After intensive washing, samples were suspended in PBS containing $0.5 \mu \mathrm{g} / \mathrm{mL}$ of propidium iodide (Wako Pure Chemical Industries, Ltd., Osaka, Japan) and analyzed using FACSort with CellQuest software (both from Becton Dickinson Immunocytometry Systems).

Detection of intracellular cytokine. T-line cells $\left(10^{6} /\right.$ well $)$ were stimulated in 48 -well plates with 25 $\mu \mathrm{g} / \mathrm{mL}$ of PLP95-116, $50 \mathrm{IU} / \mathrm{mL}$ of rhIL-2, or both, in the presence of APCs $\left(2.5 \times 10^{6} /\right.$ well $)$ for 48 hours. GolgiPlug (brefeldin A; PharMingen) was added for the last 10 hours of the incubation to disrupt Golgi function. The detection of intracellular IFN- $\gamma$ was performed using a Cytofix/Cytoperm kit (PharMingen). Briefly, cells preincubated with Fc Block ${ }^{\circledR}$ were stained with PE-labeled anti-CD4 for 30 minutes, then fixed and permeabilized with Cytofix/Cytop$\mathrm{erm}^{\circledR}$ solution for 20 minutes. The permeabilized cells were stained with FITC-labeled anti-mouse IFN$\gamma$ (XMG1.2) or isotype-matched rat IgG1 (R3-34) for 30 minutes. Samples were analyzed by flow cytometry as described above.

Histology. Four to 6 days after cell transfer, the moribund HLA-DR2/RAG-2-/- mice were anesthetized and 


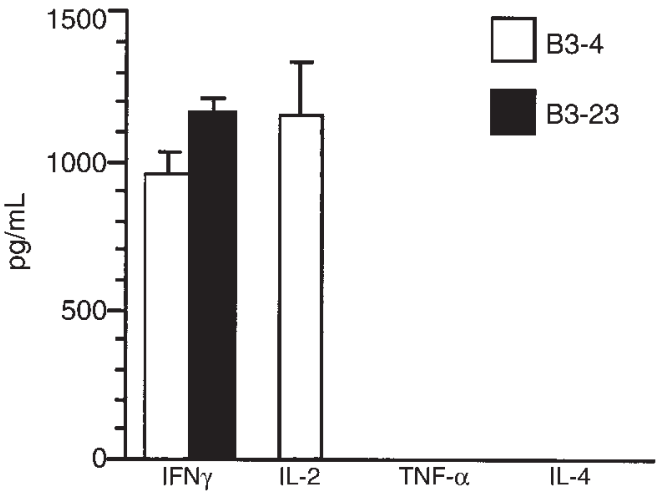

\section{Figure 3}

Cytokine production profile of TCLs B3-4 and B3-23. TCLs B3-4 and B3-23 were cultured for 48 hours with or without PLP95-116 in the presence of syngeneic splenocytes, and the culture supernatants were collected. Shown are the levels of IFN- $\gamma$, IL-2, TNF- $\alpha$, and IL- 4 detected in the supernatants of PLP95-116-stimulated cultures (no cytokine could be detected in the supernatants of the cultures without PLP95116). Data represent mean \pm SD of the values obtained by duplicate assays in 3 independent experiments.

perfused through the heart with $0.1 \mathrm{M}$ phosphate buffer followed by $4 \%$ paraformaldehyde. The brains and spinal cords were removed and fixed in the same fixative for 3 days. The paraffin-embedded tissues were sectioned at a thickness of $5 \mu \mathrm{m}$. Sections were stained with hematoxylin and eosin (H\&E) or Luxol fast blue for conventional light microscopy.

\section{Results}

Expression of HLA-DR2 molecules in HLA-DR2-B6 mice. We produced HLA-DR2-B6 mice by mating DRA-B6 mice (15) with $D R B 1 * 1502-\mathrm{B} 6$ mice that were generated from $D R B 1 * 1502-\mathrm{B} 10 . \mathrm{RQB} 3$ mice (16). Previous studies had ascertained that DRA-B6 and $D R B 1 * 1502-\mathrm{B} 10 . \mathrm{RQB} 3$ mice express $\mathrm{DR} \alpha$ and $\mathrm{DR} \beta$ chains, respectively, on immunocompetent cells. It is of note that DR $\alpha$ or DR $\beta$ chain alone could not be expressed on the cell surface, but either of the chains could appear on the cell surface in the context of xenogeneic heterodimer complexed with an endogenous I-E $\beta$ or I-E $\alpha$ chain. We first analyzed expression of DR $\alpha$ and $\mathrm{DR} \beta$ chains on splenocytes from DRAB6, DRB1*1502-B6, and HLA-DR2-B6 mice. The cells were stained with PE-labeled anti-HLA-DR $\alpha$ (L243) or FITC-labeled anti-HLA-DR $\beta \mathrm{mAb}$ (TÜ36) for single-color analysis (Figure 1a). Consistent with a previous report (17), a proportion of the splenocytes from $D R A$-B6 mice $(18.3 \pm 1.0 \% ; n=3)$ were stained with the anti-HLA-DR $\alpha \mathrm{mAb}$, indicating that the $D R \alpha$ chain appears on the cell surface in the form of a $\mathrm{DR} \alpha / \mathrm{I}-\mathrm{E} \beta^{\mathrm{b}}$ heterodimer. In contrast, $\mathrm{DR} \beta^{+}$cells were not detected in $D R B 1 * 1502-\mathrm{B} 6$ mice. This is explained by the fact that mice with a $\mathrm{B} 6$ background lack endogenous I-E $\alpha$, which is necessary for formation of a xenogeneic heterodimer. On the other hand, the splenocytes from $H L A-D R 2-\mathrm{B} 6$ mice were stained not only with the anti-HLA-DR $\alpha \mathrm{mAb}$, but also with the anti-HLA-DR $\beta \mathrm{mAb}$, implying that the DR $\beta$ chain is probably expressed in the form of a $\mathrm{DR} \alpha / \mathrm{DR} \beta$ heterodimer (HLA-DR2 molecule). It is also noteworthy that the proportion of the cells that stained brightly with the anti-HLA-DR $\alpha \mathrm{mAb}$ (M2 in Figure 1a) was significantly higher in samples from HLA-DR2-B6 mice than from DRA-B6 mice (15.1 \pm $2.8 \%, n=6$ vs. $3.9 \pm 0.3 \%, n=3$, respectively). In contrast, the expression level of endogenous I- $\mathrm{A}^{\mathrm{b}}$ molecules in the transgenic mice was not altered by $D R A$ or $D R B 1 * 1502$ transgene (data not shown). The augmentation of $\mathrm{DR} \alpha$ chain expression together with the expression of DR $\beta$ chain indicates that HLA-DR2 molecules are formed and expressed in HLA-DR2-B6 mice. Two-color analysis of HLA-DR2-B6 splenocytes (Figure 1b) further confirmed coexpression of DR $\alpha$ and DR $\beta$ chains in $32.0 \pm 5.2 \%(n=5)$ of the splenocytes. These data imply that HLA-DR2-B6 mice express 3 different MHC class II molecules: HLADR2, DR $\alpha / \mathrm{I}-\mathrm{E} \beta^{\mathrm{b}}$, and I- $\mathrm{A}^{\mathrm{b}}$.

Characterization of PLP95-116-specific TCLs generated from HLA-DR2-B6 mice. Preliminary experiments have revealed that HLA-DR2-B6 mice mount a T-cell response to PLP95-116 after immunization with the peptide (data not shown), and that the response is further augmented if the mice are pretreated with anti-NK cell antibody (anti-NK1.1 mAb, PK136). However, the primary $T$ cells that are reactive to the PLP peptide are highly heterogeneous. To focus on the HLA-DR2-restricted T cells, we attempted to generate TCLs recognizing PLP95-116 in the context of HLA-DR2 molecules. More than 100 TCLs were generated from PLP95-116-primed HLA-DR2-B6 mice, and were examined for reactivity to PLP95-116 in a proliferation assay on day 14 . We selected 15 TCLs that were reactive to PLP95-116, and restimulated the lines with the peptide for further expansion. We selected 5 PLP95-116-specific TCLs that proliferated in response to PLP95-116 in the presence of control $\mathrm{mAb}$ but not in the presence of anti-HLA-DR $\mathrm{mAb}$ (G46-6) (data not shown). Two of the 5 TCLs, designated B3-4 and B3-23, were studied in more detail.

The specificity and MHC restriction of TCLs B3-4 and TCL B3-23 are shown in Figure 2. Both of these TCLs proliferated significantly in response to PLP95-116, but

Table 1

Induction of encephalitis by TCL B3-23 in HLA-DR2/RAG-2-/- mice

\begin{tabular}{lcccc}
\cline { 2 - 2 } & Strain & Incidence & Onset day & Mortality \\
B3-23 & HLA-DR2/RAG-2-/- & $4 / 7$ & $4.0(3-5)$ & $4 / 7^{\mathrm{A}}$ \\
B3-23 & RAG-2-/- $^{-1}$ & $0 / 6$ & - & $0 / 6$ \\
B3-4 & HLA-DR2/RAG-2-/- & $0 / 8$ & - & $0 / 8$
\end{tabular}

Encephalitogenicity of TCLs B3-4 and B3-23 was assessed by adoptive transfer of T-line cells into HLA-DR2/RAG-2-/- or RAG-2-/- mice, as described in Methods. The HLA-DR2/RAG-2 ${ }^{-/-}$and $R A G-2^{-/-}$mice were monitored for the appearance of neurological signs for 8 weeks and 3 weeks, respectively. ATwo moribund mice sacrificed for histological examination are included. 
not in response to MBP143-168 in the presence of irradiated syngeneic (HLA-DR2-B6) splenocytes; these proliferative responses were almost completely blocked with the anti-HLA-DR mAb. Because splenocytes from $H L A-D R 2-\mathrm{B} 6$ mice express DR $\alpha / \mathrm{I}-\mathrm{E} \beta^{\mathrm{b}}$ and $\mathrm{I}-\mathrm{A}^{\mathrm{b}}$ molecules in addition to HLA-DR2 molecules, there still remained a possibility that PLP95-116 was presented in the context of these non-HLA-DR2 molecules. However, this possibility was excluded because $D R A$-B6 splenocytes expressing DR $\alpha / \mathrm{I}-\mathrm{E} \beta^{\mathrm{b}}$ and $\mathrm{I}-\mathrm{A}^{\mathrm{b}}$ molecules could not present the peptide to the TCLs.

Analysis by flow cytometry revealed that both of the TCLs were $\mathrm{CD}^{+} \mathrm{CD}^{-}$and expressed high levels of LFA$1, \mathrm{CD} 44$, and ICAM-1. In contrast, $\alpha 4$ integrin was moderately positive in TCL B3-4, but rarely detected in TCL B3-23. L-selectin was not detected in either of the TCLs. TCL B3-4 expressed V $\beta 2$ and V $\beta 14$ TCRs predominantly, whereas TCL B3-23 was enriched in V $\beta 6$ and $V \beta 14$-expressing $T$ cells (data not shown).

As shown in Figure 3, TCL B3-4 produced a large amount of IFN- $\gamma$ and IL-2, whereas TCL B3-23 produced IFN- $\gamma$ but not IL- 2 . Neither TNF- $\alpha$ nor IL-4 was detected in the supernatants. These results indicate that these TCLs are composed of Th1-type T cells.

To confirm HLA-DR2 restriction of the TCLs, PBMCs from healthy subject MN (HLA-DR2 $[D R B 1 * 1502] / 4)$ and YM $(H L A-D R 1 / 8)$ were used as APCs in the assays. These TCLs did not show a significant proliferative response to PLP95-116 in the presence of the human PBMCs (data not shown). In contrast, they produced comparable levels of IFN- $\gamma$ in response to PLP95-116 only when HLA-DR2-expressing PBMCs from MN were used as APCs; their IFN- $\gamma$ production was completely blocked by the anti-HLA$\mathrm{DR} \mathrm{mAb}$ (Figure 4). We further analyzed the TCLs for intracellular IFN- $\gamma$ synthesis in response to PLP95-116. As shown in Figure 5, the vast majority ( 95\%) of CD $4^{+}$ T-line cells from TCLs B3-4 and B3-23 produced high levels of IFN- $\gamma$ in response to PLP95-116 when syngeneic (HLA-DR2-B6) splenocytes were used as APCs. In contrast, when we used human PBMCs from the HLA-DR2 ${ }^{+}\left(\mathrm{DRB} 1^{*} 1502^{+}\right)$subject $(\mathrm{MN})$, IFN- $\gamma$ production was much weaker - only about $20 \%$ of the cells were defined as positive. It is possible that the poor response was due to a partial species barrier between murine accessory or costimulatory molecules and the human ligands. Therefore, we performed the assays in the presence of exogenous rhIL-2. We found that the T-line cells vigorously produced IFN- $\gamma$ with positive staining for approximately $80 \%$ of the cells. This indicates that the large majority of the line cells would recognize PLP95-116 presented by human APCs expressing HLA-DR2 molecules. Furthermore, this analysis excluded the possibility that each TCL comprises 2 subpopulations: 1 that responds to mouse APCs presenting PLP95-116, and another that is responsive to human APCs presenting PLP95-116.

Induction of autoimmune encephalitis by an HLADR2-restricted, PLP95-116-specific TCL. We next examined encephalitogenicity of the TCLs in adoptive transfer experiments. We have previously revealed that RAG-2-/- mice lacking T cells, B cells, and NKT cells become highly prone to passive experimental autoimmune encephalomyelitis (EAE) after eliminating NK cells in vivo (18). To enhance the chance of disease induction by the TCLs, we generated HLA-DR2/RAG$2^{-/-}$mice that are MHC-compatible with the TCLs and could serve as their recipients. After in vitro stimulation with PLP95-116 in the presence of syngeneic (HLA-DR2-B6) splenocytes for 3 days, $5 \times 10^{6} \mathrm{~T}$-line cells were intravenously transferred to the mice that had been injected with anti-NK1.1 mAb (PK136) 1 day before cell transfer. TCL B3-4 did not induce any neurological sign in the recipient mice during the observation period of 8 weeks (Table 1). In contrast, 4 of the 7 mice receiving TCL B3-23 developed neurological a

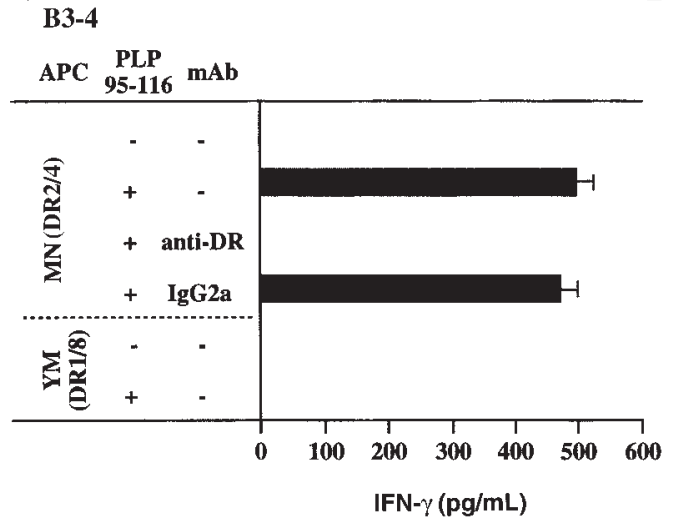

b

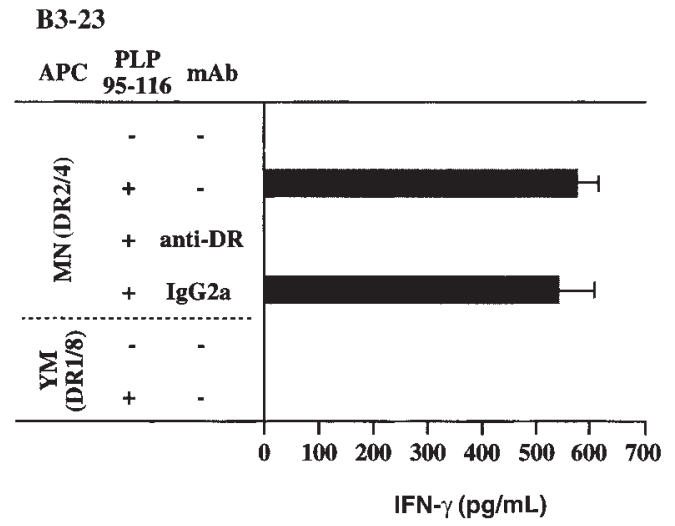

Figure 4

IFN- $\gamma$ production by TCLs B3-4 and B3-23 in the presence of human PBMCs. TCLs B3-4 (a) and B3-23 (b) were cultured for 48 hours with or without PLP95-116 in the presence of human PBMCs from subject MN (HLA-DR2 [DRB1*1502]/4) or YM ([HLA-DR1]/8), and the supernatants were collected for measurement of IFN- $\gamma$. Blocking effects of anti-HLA-DR mAb (G46-6) or isotype-matched control IgG2a (G155178) were also examined as indicated. Data represent mean \pm SD of the values obtained by duplicate assays in 4 independent experiments. 


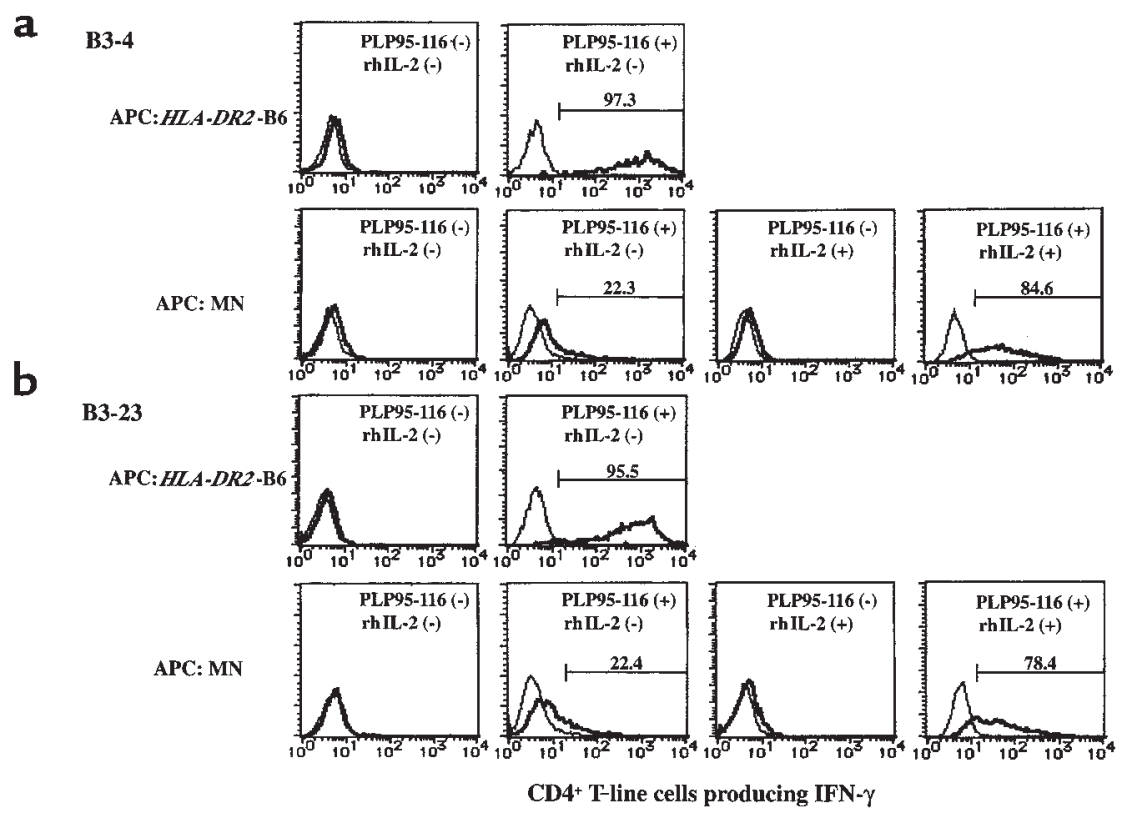

\section{Figure 5}

Flow cytometric detection of intracellular IFN- $\gamma$ after stimulation with PLP95-116. Intracellular IFN- $\gamma$ synthesis of TCLs responding to PLP95-116 was examined using different sources of APCs: syngeneic splenocytes from HLA-DR2-B6 mice, or human PBMCs (from subject MN expressing HLA-DR2 [DRB1*1502]/4). The line cells B3-4 (a) and B3-23 (b) were incubated for 48 hours in the presence of the APCs with (+) or without (-) PLP95-116 $(25 \mu \mathrm{g} / \mathrm{mL})$ and rhIL-2 $(25 \mu \mathrm{g} / \mathrm{mL})$. GolgiPlug ${ }^{\circledR}$ (brefeldin A) was added for the last 10 hours of incubation, and the cells were stained for intracellular IFN- $\gamma$ as described in Methods. Only the CD4 ${ }^{+}$T-cell population was gated for analysis, and the frequency (\%) of the IFN$\gamma^{+}$population is shown (above bars). Note that exogenous rhIL-2 markedly enhanced IFN- $\gamma$ production in the presence of the human APCs.

signs of cerebellar ataxia 3-5 days after transfer. Signs of EAE in the mice began with the turning of their heads and trunks to 1 side and progressed to axial rotatory movement. Two mice died 1 day after clinical onset. Because the other 2 symptomatic mice developed a comparable progression of disease, we sacrificed them for histological examination 1 day after clinical onset. It was of note that there was no sign of the tail atony or hind-limb paresis that is characteristic of conventional EAE models. In contrast, transfer of the pathogenic TCL into nontransgenic $R A G-2^{-/-}$ mice did not induce any clinical sign during the 3week observation period. Based on these results, we presume that HLA-DR2-restricted recognition of endogenous PLP95-116 in vivo lay behind the neurological sign induced by TCL B3-23.

To confirm the CNS involvement, we performed pathological studies of the 2 moribund HLA-DR2/RAG$2^{-/-}$mice receiving TCL B3-23. Examination of the CNS samples from these mice revealed meningeal and perivascular parenchymal inflammatory infiltrates (Figure 6, c-e, g) composed of mononuclear cells and neutrophils (Figure $6 \mathrm{~h}$ ). Infiltration was most prominent in the cerebrum and cerebellum. In addition, discrete inflammatory demyelinating lesions were scattered predominantly in the deep cerebral cortex (Figure 6 , a, b, e, and f). By contrast, only very low levels of meningeal infiltrates were detected in the spinal cord. These histological findings were thought to correlate well with the clinical phenotype.

\section{Discussion}

We previously reported that the frequency of PLP95116-specific T cells is significantly elevated in HLA$\mathrm{DR} 2^{+} \mathrm{MS}$ patients in comparison with those who are HLA-DR2- (6). The association of PLP95-116 with HLA-DR2 ${ }^{+}$MS was later confirmed by Trotter et al. (10). Using whole PLP molecules, they further defined PLP95-117 as an immunodominant epitope for HLA-DR2 ${ }^{+}$MS. More recently, we reported evidence for transient or continuous activation/expansion of PLP95-116-specific T-cell clones in an HLADR2 ${ }^{+}$(DRB1*1501/DRB1*1502+) MS patient (19). Despite these observations, the in vivo role of PLP95-116-specific $\mathrm{T}$ cells has remained speculative. Here we demonstrate for the first time that HLADR2-restricted, (DRB1*1502-restricted) PLP95116-specific $T$ cells can cause autoimmune encephalitis in mice expressing HLA-DR2 (DRB1*1502) molecules. Because humans and rodents share identical amino acids in PLP95-116 residues (20), our results indicate that PLP95-116 can be a target epitope for autopathogenic $\mathrm{T}$ cells not only in HLA-DR2 $(D R B 1 * 1502)$ transgenic mice but also in HLA-DR2 ${ }^{+}\left(\mathrm{DRB} 1 * 1502^{+}\right)$MS. Recent studies have demonstrated that transgenic mice expressing human HLA-DR molecules could develop encephalomyelitis after being challenged with myelin extract or peptide $(21,22)$. However, as encephalitogenic, DR-restricted TCLs or clones were not established from the transgenic mice, it 
remained unclear if HLA-DR-restricted T cells alone could induce encephalomyelitis, or if other cell types such as B cells are necessary.

A number of studies have revealed that susceptibility to MS in Caucasian populations is positively linked with the $D R B 1 * 1501$ allele (23). However, our study has revealed that a proportion of Japanese MS patients possess DRB1*1502 but not DRB1*1501, and that TCLs recognizing the DRB1*1501-related MBP or PLP peptides can be efficiently generated from DRB1* $1502^{+}$patients (Ohashi et al., unpublished data). Furthermore, DRB1*1502 was found to be present in the vast majority of patients with concentric sclerosis (Baló's disease) (unpublished observation), which is probably a variant form of MS (24). Moreover, DRB1*1501 and DRB1*1502 differ only in the amino acid at position 86 of the DR $\beta$ chain (valine in DRB1*1501 and glycine in DRB1*1502) (25). Although the size of the P1 pocket in the peptide-binding groove differs between the DRB $1 * 1501$ and DRB1*1502 molecules (26, 27), MBP85-99 peptide could be presented to HLA-DR2-restricted (DRB1*1501-restricted) T cells also in the context of HLA-DR2 (DRB1*1502) molecules (28). These observations encouraged us to use HLA-DR2 (DRB1*1502) transgenic mice for establishing a model for HLADR2 ${ }^{+}$MS, or at least for relevant subtypes of MS.

It is of particular interest that the clinical and pathological features of EAE described herein are quite different from those of classical EAE. Whereas the classical EAE models are characterized by ascending paralysis caused by the lesions in the spinal cord white matter, the neurological sign in the HLADR2/RAG-2-/- mice receiving TCL B3-23 was represented by ataxia without paralysis, consistent with the predominant lesions in the cerebrum and cerebellum. Regarding the unique EAE phenotype in this study, it is relevant that some EAE models induced by PLP peptides exhibit similar clinical phenotypes, characterized by rotatory movement or ataxia (29-31). Just such an unusual form of EAE, displaying ataxia, was seen by Greer and colleagues in $\mathrm{BALB} / \mathrm{c}, \mathrm{CBA} / \mathrm{J}$, and $\mathrm{C} 3 \mathrm{H} / \mathrm{HeJ}$ mice when PLP peptides within PLP residues 178-232 used for active immunization, whereas encephalitogenic peptides within other PLP domains caused a typical form of EAE (31). Based on these results, Greer et al. speculated that there might be a correlation between target epitope and disease phenotype. Another relevant piece of information is that $\mathrm{T}$ cells recognizing MBP, a representative myelin antigen, and $\mathrm{T}$-cells specific for a non-myelin antigen, $S 100 \beta$ protein, have been shown to cause distinct disorders in the rat model of EAE (32). Very interestingly, S100ß-specific T cells invaded not only white matter but also brain cortex, whereas MBP-specific T cells are primarily a cause of white matter lesions. Because distribution of MBP and $\mathrm{S} 100 \beta$ within the CNS could not account for this difference,Kojima et al explained it by speculating that there is a differing capacity to process and present specific antigen/epitope among different regions of the CNS. These reports together with our own data suggest that a variety of neurological signs of MS may be finely dissected after identifying predominant target epitopes in each patient. In this regard, induction of cerebral cortical lesions induced by HLA-DR2-restricted, PLP95-116-specific T cells may be relevant to understanding why cortical lesions are seen in some MS patients but not in others (33). Further exploration of the correlation between epitope and disease phenotype in HLA-DR2 (DRB1*1502) transgenic mice may provide essential information for understanding anti-myelin autoimmunity.
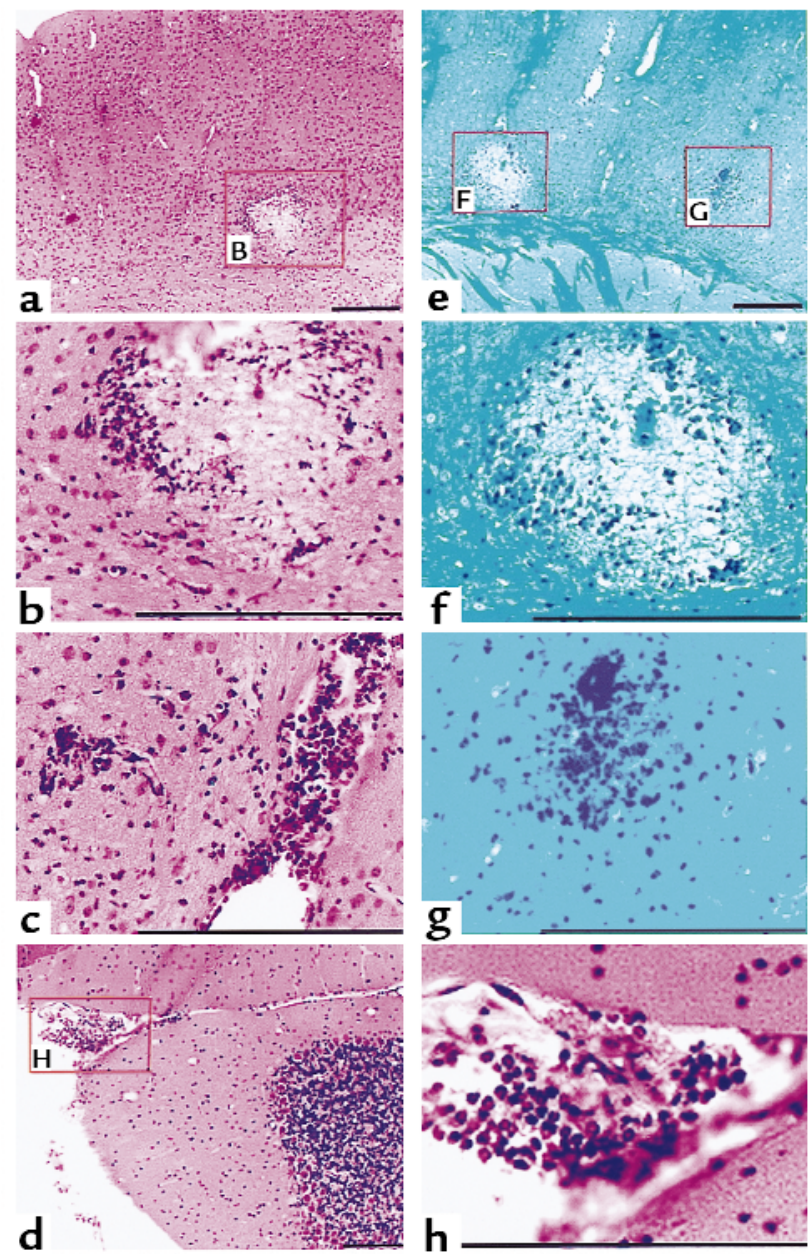

Figure 6

Histology of CNS sections from moribund HLA-DR2/RAG-2-/- mice receiving TCL B3-23. Shown are representative inflammatory foci in the cerebrum and cerebellum from mice receiving TCL B3-23. (a-d and $\mathbf{h}$ ) H\&E staining. (e, $\mathbf{f}$, and $\mathbf{g}$ ) Luxol fast blue staining. Bars $=100$ $\mu \mathrm{m}$. Higher magnifications of the boxed areas $B, H, F$, and $G$ are illustrated in $\mathbf{b}, \mathbf{h}, \mathbf{f}$, and $\mathbf{g}$, respectively. The inflammatory lesions include perivascular infiltrates in the cerebral cortex $(\mathbf{g})$ and periventricular white matter (c) as well as inflammatory infiltrates within the ventricle (c) and cerebellar meninges (d). These infiltrates were primarily composed of mononuclear cells, but neutrophils were also identified (h). Some of the inflammatory foci located in the deep cerebral cortex (a and $\mathbf{e}$ ) were accompanied by demyelinating lesions ( $\mathbf{b}$ and $\mathbf{f}$ ). 


\section{Acknowledgments}

This work was supported by a Research on Brain Science grant from the Ministry of Health and Welfare of Japan, and a grant from the Science and Technology Agency of Japan. The authors thank Keikichi Takahashi for help with the genetic analysis of the transgenic mice.

1. Martin, R., McFarland, H.F., and McFarlin, D.E. 1992. Immunological aspects of demyelinating diseases. Annu. Rev. Immunol. 10:153-187.

2. Hafler, D.A., and Weiner, H.L. 1995. Immunologic mechanisms and therapy in multiple sclerosis. Immunol. Rev. 144:75-107.

3. Zhang, J.W., et al. 1994. Increased frequency of interleukin 2-responsive $\mathrm{T}$ cells specific for myelin basic protein and proteolipid protein in peripheral blood and cerebrospinal fluid of patients with multiple sclerosis. J. Exp. Med. 179:973-984.

4. Allegretta, M., Nicklas, J.A., Sriram, S., and Albertini, R.J. 1990. T cells responsive to myelin basic protein in patients with multiple sclerosis. Science. 247:718-721

5. Trotter, J.L., et al. 1997. HPRT mutant T-cell lines from multiple sclerosis patients recognize myelin proteolipid peptides. J. Neuroimmunol. 75:95-103.

6. Ohashi, T., et al. 1995. Analysis of proteolipid protein (PLP) in multiple sclerosis: identification of PLP95-116 as an HLA-DR2,w15-associated determinant. Int. Immunol. 7:1771-1778.

7. Oksenberg, J.R., et al. 1993. Selection for T-cell receptor V $\beta$-D $\beta$-J $\beta$ gene rearrangements with specificity for a myelin basic protein peptide in brain lesions of multiple sclerosis. Nature. 362:68-70.

8. Kondo, T., et al. 1995. TCR repertoire to proteolipid protein (PLP) in multiple sclerosis (MS): homologies between PLP-specific T cells and MS-associated T cells in TCR junctional sequences. Int. Immunol. 8:123-130.

9. Ota, K., et al. 1990. T-cell recognition of an immunodominant myelin basic protein epitope in multiple sclerosis. Nature. 346:183-187.

10. Trotter, J.L., et al. 1998. T cell recognition of myelin proteolipid protein and myelin proteolipid protein peptides in the peripheral blood of multiple sclerosis and control subjects. J. Neuroimmunol. 84:172-178.

11. Moalem, G., et al. 1999. Autoimmune T cells protect neurons from secondary degeneration after central nervous system axotomy. Nat. Med. 5:49-55.

12. Kerschensteiner, M., et al. 1999. Activated human T cells, B cells, and monocytes produce brain-derived neurotrophic factor in vitro and in inflammatory brain lesions: a neuroprotective role of inflammation? J. Exp. Med. 189:865-870.

13. Besser, M., and Wank, R. 1999. Clonally restricted production of the neurotrophins brain-derived neurotrophic factor and neurotrophin-3 mRNA by human immune cells and Th1/Th2-polarized expression of their receptors. J. Immunol. 162:6303-6306.

14. Shinkai, Y., et al. 1992. RAG-2-deficient mice lack mature lymphocytes owing to inability to initiate V(D)J rearrangement. Cell. 68:855-867.
15. Fukui, Y., et al. 1993. T-cell repertoire in a strain of transgenic C57BL/6 mice with the HLA-DRA gene on the X-chromosome. Immunogenetics. 37:204-211

16. Gonzalez-Gay, M.A., et al. 1996. Human leukocyte antigen-DRB1*1502 (DR2Dw12) transgene reduces incidence and severity of arthritis in mice. Hum. Immunol. 50:54-60.

17. Lawrence, S.K., et al. 1989. HLA-DR $\alpha$ faithfully reconstitutes IE-controlled immune functions and induces cross-tolerance to $\mathrm{E} \alpha$ in $\mathrm{E} \alpha^{0}$ mutant mice. Cell. 58:583-594.

18. Zhang, B.N., Yamamura, T., Kondo, T., Fujiwara, M., and Tabira, T. 1997. Regulation of experimental autoimmune encephalitis by natural killer (NK) cells. J. Exp. Med. 186:1677-1687.

19. Illés, Z., et al. 1999. Identification of autoimmune $T$ cells among in vivo expanded CD25 ${ }^{+} \mathrm{T}$ cells in multiple sclerosis. J. Immunol. 162:1811-1817.

20. Tuohy, V.K. 1994. Peptide determinants of myelin proteolipid protein (PLP) in autoimmune demyelinating disease: a review. Neurochem. Res. 19:935-944.

21. Das, P., et al. 1997. HLA transgenic mice as humanized models of multiple sclerosis (MS): studies on EAE. Hum. Immunol. 55(Suppl. 1):4. (Abstr.)

22. Ito, K., et al. 1996. HLA-DR4-IE chimeric class II transgenic, murine class II-deficient mice are susceptible to experimental allergic encephalomyelitis. J. Exp. Med. 183:2635-2644.

23. Olerup, O., and Hillert, J. 1991. HLA class II-associated genetic susceptibility in multiple sclerosis: a critical evaluation. Tissue Antigens. 38:1-15.

24. Yao, D.L., et al. 1994. Concentric sclerosis (Balo): morphmetric and in situ hybridization study of lesions in six patients. Ann. Neurol. 35:18-30.

25. Marsh, S.G.E., and Bodmer, J.G. 1993. HLA class II nucleotide sequences, 1992. Immunogenetics. 37:79-94.

26. Wucherpfennig, K.W., and Strominger, J.L. 1995. Selective binding of self peptides to disease-associated major histocompatibility complex (MHC) molecules: a mechanism for MHC-linked susceptibility to human autoimmune diseases. J. Exp. Med. 181:1597-1601.

27. Smith, K.J., Pyrdol, J., Gauthier, L., Wiley, D.C., and Wucherpfennig, K.W. 1998. Crystal structure of HLA-DR2 (DRA*0101, DRB1*1501) complexed with a peptide from human myelin basic protein. J. Exp. Med. 188:1511-1520

28. Wucherpfennig, K.W., and Strominger, J.L. 1995. Molecular mimicry in $T$ cell-mediated autoimmunity: viral peptides activate human $T$ cell clones specific for myelin basic protein. Cell. 80:695-705.

29. Sobel, R.A., Greer, J.M., and Kuchroo, V.K. 1994. Minireview: autoimmune responses to myelin proteolipid protein. Neurochem. Res. 19:915-921.

30. Endoh, M., et al. 1986. DM-20, a proteolipid apoprotein, is an encephalitogen of acute and relapsing autoimmune encephalomyelitis in mice. J. Immunol. 137:3832-3835.

31. Greer, J.M., et al. 1996. Immunogenic and encephalitogenic epitope clusters of myelin proteolipid protein. J. Immunol. 156:371-379.

32. Kojima, K., et al. 1994. Experimental autoimmune panencephalitis and uveoretinitis transferred to the Lewis rat by T lymphocytes specific for $\mathrm{S} 100 \beta$ molecule, a calcium binding protein of astroglia. J. Exp. Med. 180:817-829.

33. Kidd, D., et al. 1999. Cortical lesions in multiple sclerosis. Brain. 122:17-26. 